\title{
Characterisation of utrophin modulator SMT C1100 as a non-competitive inhibitor of firefly luciferase
}

\author{
Isabel V.L. Wilkinson ${ }^{\mathrm{a}}$, Jessica K. Reynolds ${ }^{\mathrm{a}}$, Sébastien R.G. Galan ${ }^{\mathrm{a}}$, Aini Vuorinen ${ }^{\mathrm{a}}$, \\ Adam J. Sills $^{\mathrm{a}}$, Elisabete Pires $^{\mathrm{a}}$, Graham M. Wynne ${ }^{\mathrm{a}}$, Francis X. Wilson ${ }^{\mathrm{b}}$, Angela J. Russell ${ }^{\mathrm{a}, \mathrm{c}, *}$ \\ ${ }^{a}$ Department of Chemistry, University of Oxford, Chemistry Research Laboratory, Mansfield Road, Oxford OX1 3TA, UK \\ ${ }^{\mathrm{b}}$ Summit Therapeutics plc, 136a Eastern Avenue, Milton Park, Abingdon, Oxfordshire OX14 4SB, UK \\ ${ }^{\mathrm{c}}$ Department of Pharmacology, University of Oxford, Mansfield Road, Oxford OX1 3PQ, UK
}

\section{A R T I C L E I N F O}

\section{Keywords:}

Firefly luciferase

Bioluminescence

Assay interference

Enzyme inhibitor

Inhibition mechanism

Enzyme kinetics

Photoaffinity probe

Enzyme mechanism

Duchenne muscular dystrophy

\begin{abstract}
A B S T R A C T
Firefly luciferase (FLuc) is a powerful tool for molecular and cellular biology, and popular in high-throughput screening and drug discovery. However, FLuc assays have been plagued with positive and negative artefacts due to stabilisation and inhibition by small molecules from a range of chemical classes. Here we disclose Phase II clinical compound SMT C1100 for the treatment of Duchenne muscular dystrophy as an FLuc inhibitor $\left(\mathrm{K}_{\mathrm{D}}\right.$ of $0.40 \pm 0.15 \mu \mathrm{M})$. Enzyme kinetic studies using SMT C1100 and other non-competitive inhibitors including resveratrol and NFkBAI4 identified previously undescribed modes of inhibition with respect to FLuc's luciferyl adenylate intermediate. Employing a photoaffinity strategy to identify SMT C1100's binding site, a photolabelled SMT C1100 probe instead underwent FLuc-dependent photooxidation. Our findings support novel binding sites on FLuc for non-competitive inhibitors.
\end{abstract}

\section{Introduction}

Firefly luciferase (FLuc) is widely used in academia and industry due to its excellent sensitivity and dynamic range, and its ease of use [1]. Firefly luciferase (Photinus pyralis) is bioluminescent, enabling sensitive detection because a light excitation source is not required, reducing the background signal [2]. Dynamic changes in luciferase abundance can be detected due to its short half-life of 3-4 hrs in mammalian cells [3]. FLuc is used in a broad range of often highthroughput applications including reporter gene assays, in vivo imaging, cell viability assays, cAMP sensing for cell signalling assays and more [2].

However, FLuc assays are sensitive to positive and negative artefacts arising from compound interferences [1]. Studies conducted by Auld et al. have shown that $\sim 5 \%$ of compounds in large libraries interfere with FLuc at $11 \mu \mathrm{M}$ [4], while $\sim 6 \%$ of the GSK published protein kinase inhibitor set are FLuc inhibitors [5]. Furthermore, up to $98 \%$ of compounds identified as high throughput screen (HTS) hits from FLuc reporter assays have been found to be FLuc inhibitors [6,7]. Many commonly used tool compounds such as p53-inhibitor pifithrin- $\alpha$ [8], resveratrol [9] and aryl hydrocarbon receptor inhibitor $\beta$-naphthoflavone [10] have also been found to be FLuc inhibitors. Consequently, hit and tool compounds should be routinely assessed for FLuc inhibition to validate interpretation of results from FLuc assays.

FLuc interferences are typically due to direct binding of the compound to luciferase rather than through light attenuation or scattering $[4,11]$. Compound binding has been found to impart a thermal stabilisation on the FLuc enzyme, which increases its half-life independently of transcription or translation $[3,12]$. This stabilisation effect is amplified by the short half-life of FLuc coupled with long compound incubation times (12-48 h) typically seen with reporter gene assays [13]. Increases in luciferase abundance by compound binding can lead to false positives, while false negatives arise from inhibition of the bioluminescence reaction.

Firefly luciferase's catalytic bioluminescence reaction proceeds via a relatively well characterised multistep mechanism (Fig. 1a). FLuc binds substrates D-luciferin (D- $\left.\mathrm{LH}_{2}\right) \mathbf{1}$ and ATP to form the reactive intermediate luciferyl-adenylate ( $\mathrm{LH}_{2}$-AMP) 2 [14]. Upon adenylation, FLuc

\footnotetext{
Abbreviations: D-LH 2 , D-luciferin; DLSA, 5'-O-[ $N$-dehydroluciferyl)-sulfamoyl]adenosine; DMD, Duchenne muscular dystrophy; ESI MS/MS, Electrospray ionization

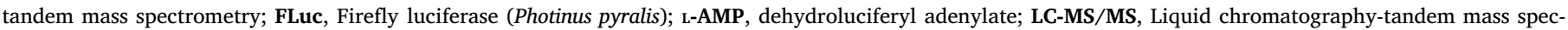

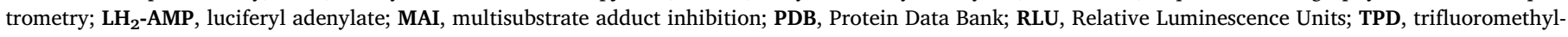
phenyl diazirine; UTR, untranslated region

* Corresponding author at: Department of Chemistry, University of Oxford, Chemistry Research Laboratory, Mansfield Road, Oxford OX1 3TA, UK.

E-mail address: angela.russell@chem.ox.ac.uk (A.J. Russell).
} 
a
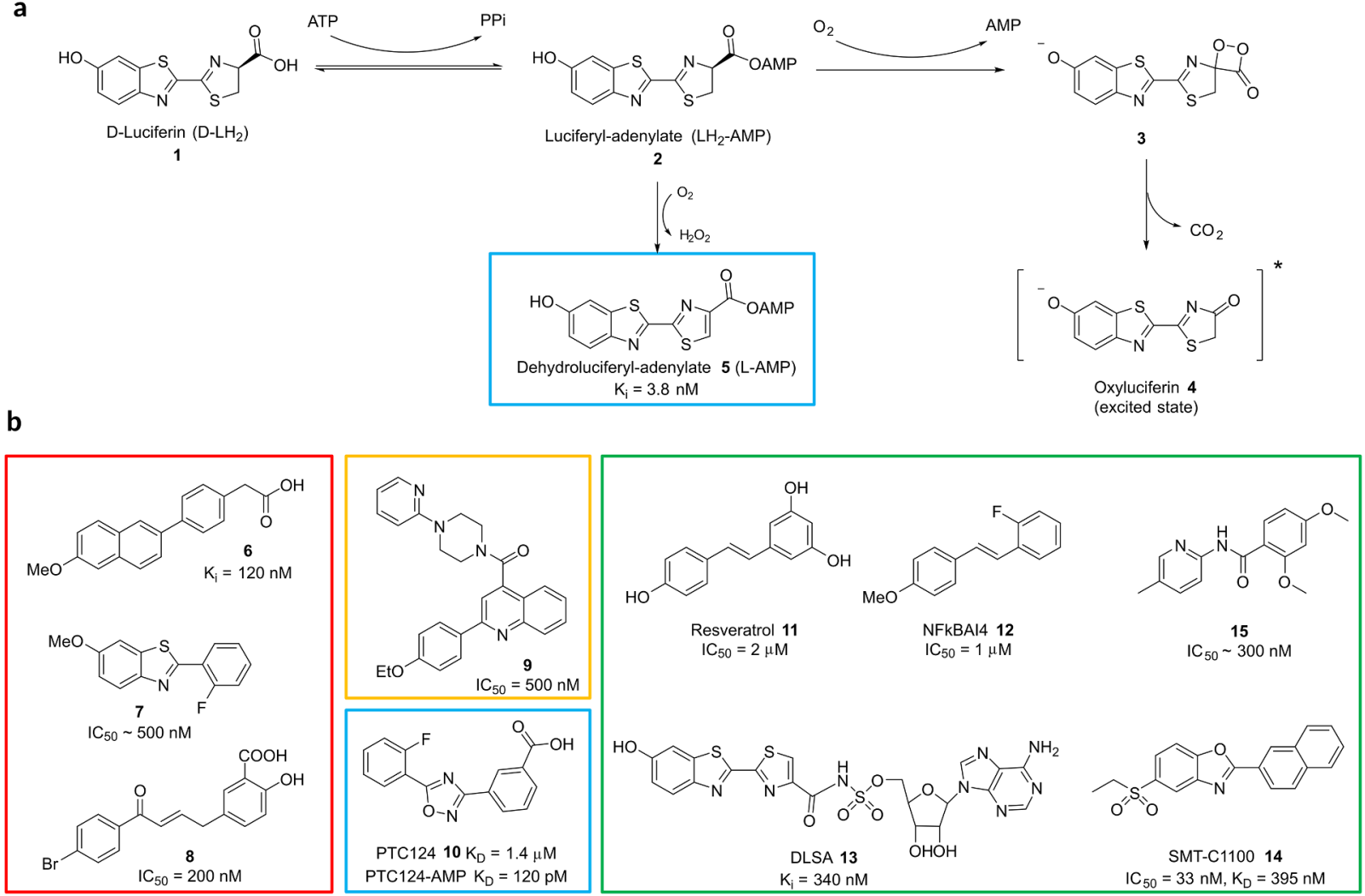

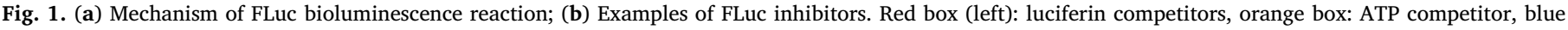
boxes: multisubstrate adduct inhibitors, green box (right): non-competitive inhibitors.

undergoes a conformational change [15]. Then, $\mathrm{LH}_{2}$-AMP reacts with molecular oxygen to form an electronically excited dioxetanone 3 which collapses to generate oxyluciferin 4 in an excited state and carbon dioxide [16]. Relaxation to the ground state of oxyluciferin releases a photon of light of wavelength 560-610 nm [16]. FLuc can also perform 'dark' reactions such as oxidation of the intermediate $\mathrm{LH}_{2}$ AMP to form the potent tight-binding inhibitor L-AMP $\mathbf{5}$ which is responsible for the flashing of firefly lanterns $[11,14,17]$.

\subsection{Mechanisms of FLuc inhibition}

FLuc inhibitors are typically linear, planar small molecules and some related structure-activity-relationships have been established in the literature $[4,11,18]$. Interestingly, inhibitors comprise a broad range of chemotypes (Fig. 1b), revealing FLuc to be a highly promiscuous enzyme. This wide structural diversity complicates prediction of compounds as FLuc inhibitors, hindering recognition of assay artefacts. It also suggests that multiple binding sites might exist on the FLuc enzyme to accommodate such a range of inhibitors.

Reported mechanisms of luciferase inhibition so far include substrate competition, multisubstrate adduct inhibition (MAI) and nonspecific unfolding [19-21]. Most common amongst the reported mechanisms are inhibitors that compete with the substrate D-luciferin (FLuc $\mathrm{K}_{\mathrm{M}} \sim 10 \mu \mathrm{M}[22,23]$ ). These include the analogues L-luciferin [24], dehydroluciferin [25], oxyluciferin [26], as well as distinct chemotypes such as fatty acids [27,28], and 2-phenylnaphthalenes 6 [29] (Fig. 1b). Three inhibitor-bound FLuc complexes have been reported from soaking of apo crystals, each of which found the inhibitor (benzothiazole derivative 7 [4], bromoform [30] and chalcone derivative 8 [31]) bound in the luciferin pocket. Computational analysis of FLuc inhibitors binding to this pocket has led to predictor tools designed to flag potential HTS false positives [32,33].

FLuc inhibitors which compete with the ATP substrate have been less frequently observed but include a series of quinoline containing compounds 9 which were also found to compete with luciferin [11]. The lower frequency of identification of inhibitors of this mechanism is likely due to high saturating intracellular concentrations of ATP $(\sim 1$ $\mathrm{mM}$ ) which masks the evidence of this inhibition mode.

Multisubstrate adduct inhibitors (MAI) are single molecules comprising two or more substrates of the enzyme [34,35]. This approach often leads to highly potent and selective inhibitors, with examples for FLuc including the naturally occuring L-AMP inhibitor and PTC124 10 (Ataluren/Translarna), both of which contain carboxylic acids and form adenylate adducts after reaction with ATP and release of pyrophosphate [36]. PTC124 has been granted conditional approval in Europe as a treatment for Duchenne muscular dystrophy in patients with nonsense mutations [37]. It was originally discovered using a cellular FLuc reporter nonsense codon assay [38] but was subsequently found to inhibit FLuc with an $\mathrm{IC}_{50}$ of $7 \mathrm{nM}$, correlating to the activity in the original assay [20].

Numerous examples of inhibitors of FLuc which are non-competitive with respect to luciferin or ATP or both exist in the literature. These include compounds such as resveratrol 11 [9] and NF-kB inhibitor 12 [39], which continue to be used in luciferase reporter assays without control for their artefactual behavior [40]. Other non-competitive inhibitors of different chemotypes have been identified with IC $_{50}$ values as low as $0.26 \mathrm{nM}[11,12,18]$. However, the binding mode of these inhibitors has not yet been reported. The $\mathrm{LH}_{2}$-AMP mimic DLSA 13 reported by Branchini et al. was found to be non-competitive with respect to the two substrates luciferin and ATP, but competitive with the $\mathrm{LH}_{2}$-AMP intermediate [41]. This was rationalised by the significant conformational change involving a $\sim 140^{\circ}$ rotation of the $C$ terminal domain that occurs after the activating adenylation reaction forming $\mathrm{LH}_{2}$-AMP, offering different binding environments within the same active site [15].

In this work, the small molecule SMT C1100 (ezutromid) 14 was 
a
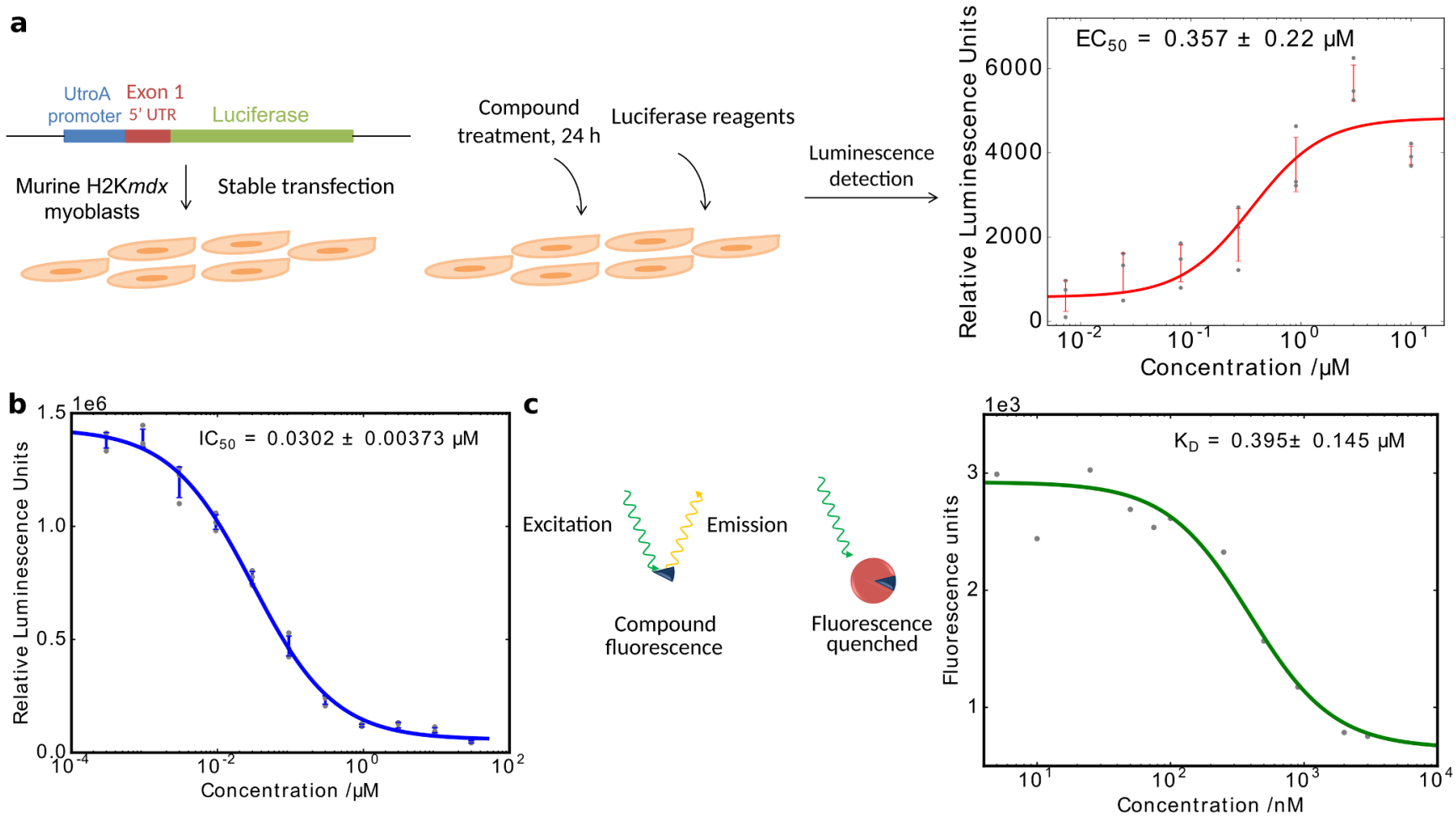

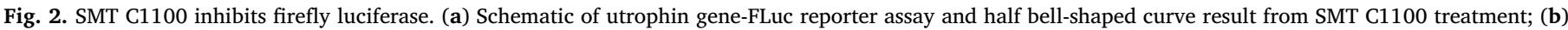

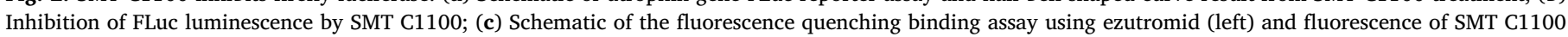
(300 nM, $317 \mathrm{~nm}$ excitation, $390 \mathrm{~nm}$ emission) measured after incubation (15 min) with firefly luciferase (5-3000 nM).

identified as a non-competitive FLuc inhibitor. It inhibits FLuc via a novel mechanism, along with other reported non-competitive inhibitors.

\section{Results and discussion}

\subsection{SMT C1100 is an FLuc inhibitor}

SMT C1100 was originally discovered using a FLuc reporter gene assay for utrophin upregulation. Although it was later shown to upregulate utrophin at both the mRNA and protein level in DMD mouse models and in human DMD patients' cells [42-44], in dose-response analyses in the original reporter gene assay it displayed a bell-shaped curve, distinctive of FLuc inhibitors which stabilise FLuc and inhibit the bioluminescence reaction at high concentrations (Fig. 2a). This led us to suspect that an interaction between ezutromid and FLuc may be at least contributing to the output. As anticipated, when subjected to an in vitro enzyme assay with recombinant FLuc, SMT C1100 was found to be a potent FLuc inhibitor, with an $\mathrm{IC}_{50}$ of $0.033 \mu \mathrm{M}$ (Fig. 2b). Since SMT $\mathrm{C} 1100$ does not absorb above $380 \mathrm{~nm}$, it cannot quench the green tone FLuc luminescence (Supplementary Fig. S1), so must be interfering with FLuc via a different mechanism. Conservation of SMT C1100's inhibition of FLuc in the presence of a range of detergents (Supplementary Fig. S2) excluded small molecule aggregation as the mechanism of inhibition [45]. SMT C1100 is however highly fluorescent (excitation at $317 \mathrm{~nm}$, emission at $390 \mathrm{~nm}$ ), so its binding affinity for FLuc could be determined via quenching of the compound's fluorescence upon binding of the protein. Fitting of the fluorescence quenching with a four parameter logistic function gave an apparent $\mathrm{K}_{\mathrm{D}}$ of $0.40 \pm 0.15 \mu \mathrm{M}$ and Hill coefficient of $0.98 \pm 0.24$ (Fig. 2c). This apparent FLuc $\mathrm{K}_{\mathrm{D}}$ correlates well with the $\mathrm{EC}_{50}$ from the FLuc reporter gene assay $(0.36 \pm 0.22 \mu \mathrm{M}$, Fig. 2a), indicating that the original result was likely biased by FLuc interference.

\subsection{SMT C1100 does not compete with luciferin or ATP}

SMT C1100's benzoxazole core and planar, hydrophobic structure are characteristic of luciferin competitors. Docking studies using the benzothiazole inhibitor 7 bound FLuc structure (PDB: 4e5d [4]) as a model supported this hypothesis (Fig. 3a), with SMT C1100 overlaying the inhibitor pose as anticipated. However, kinetic experiments varying first luciferin then ATP (with the other substrate held at saturating concentrations) indicated that substrate competition was not the mechanism by which SMT C1100 operates. Instead, fitting of initial rates to the Michaelis-Menten equation under rapid-equilibrium steady state kinetics led to determination of non-competitive inhibition with respect to luciferin, and uncompetitive with respect to ATP (Fig. 3d-e). These results were supported by Lineweaver-Burk analysis of the results, which showed FLuc's $\mathrm{K}_{\mathrm{M}}$ for luciferin unchanged at $18.2 \mu \mathrm{M}$ (close to the literature value of $16.9 \mu \mathrm{M}$ [23]) as is expected for non-competitive inhibition (Fig. 3b) and the $\mathrm{K}_{\mathrm{M}}$ for ATP decreasing as seen in uncompetitive inhibition (Fig. 3c). The $\mathrm{K}_{\mathrm{I}}$ was calculated as $0.76 \pm 0.43 \mu \mathrm{M}$ from Dixon plot analysis of the results (Supplementary Fig. S3).

To assess whether SMT C1100 could be competing with $\mathrm{LH}_{2}$-AMP for binding to FLuc's second catalytic conformation, further kinetic experiments were carried out, varying $\mathrm{LH}_{2}$-AMP which was supplied as the sole substrate (initial velocity and Lineweaver-Burk plots in Fig. 4). DLSA was used as a positive control and was confirmed to compete with $\mathrm{LH}_{2}$-AMP (Supplementary Fig. S4). SMT C1100 was found not to compete with $\mathrm{LH}_{2}$-AMP, but instead was shown to display partial mixed inhibition, meaning it binds to both the FLuc and the FLuc: $\mathrm{LH}_{2}-\mathrm{AMP}$ complex, and also does not fully inhibit light production. Other compounds that have been found to bind via mixed type inhibition have also been reported. A class of aryl triazoles were found to have mixed non-competitive inhibition with respect to substrate analogue aminoluciferin [46], while some pyrrolo[2,3-d]pyrimidines had mixed competitive inhibition with respect to luciferin [47]. To determine whether 
a

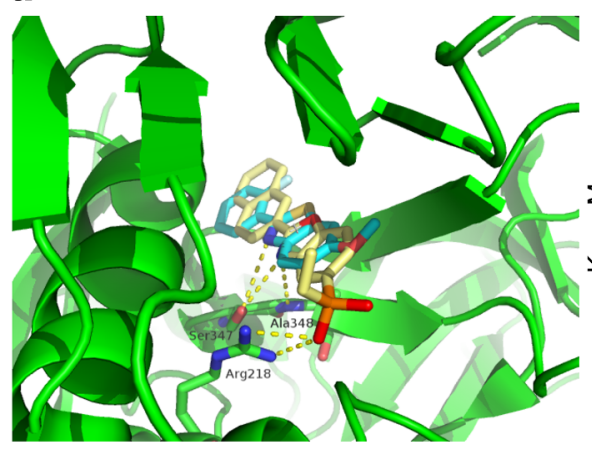

b

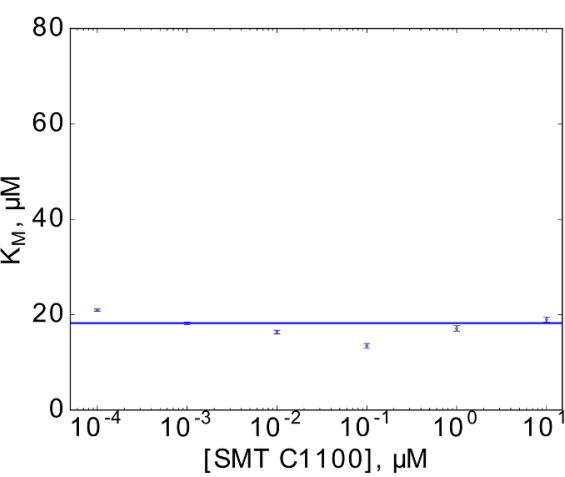

C

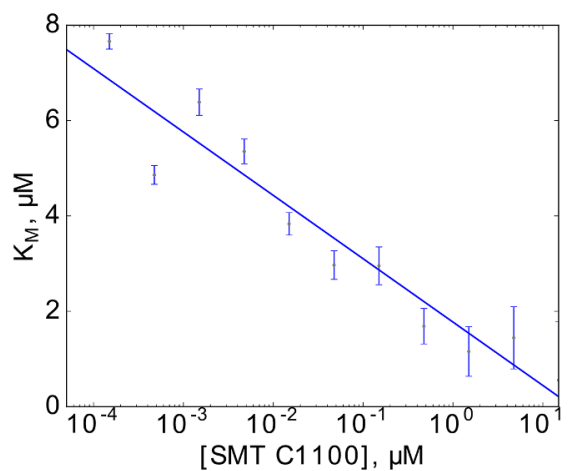

d

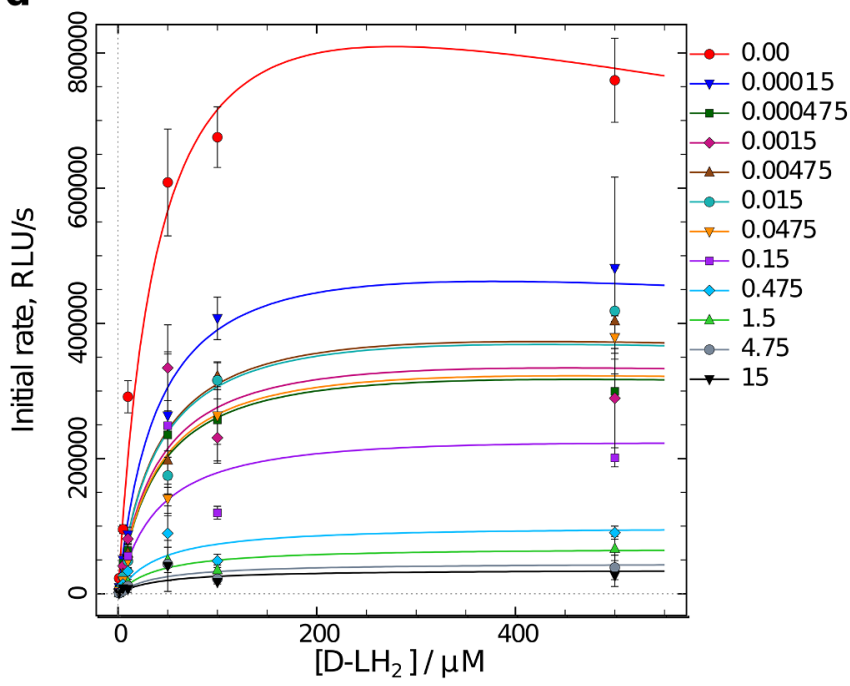

e

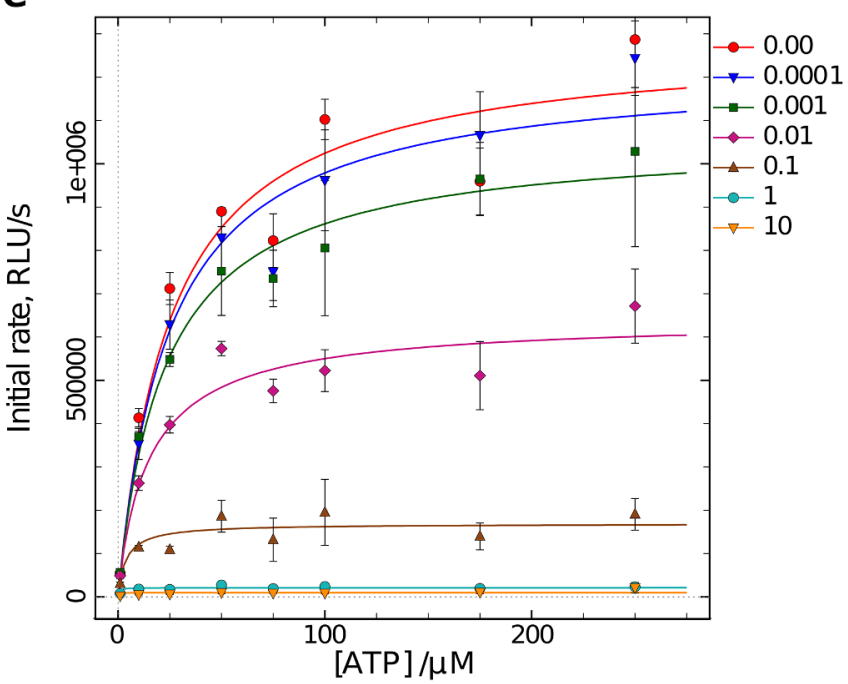

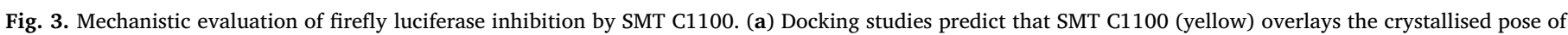

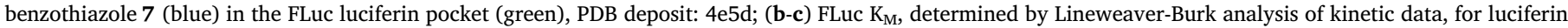

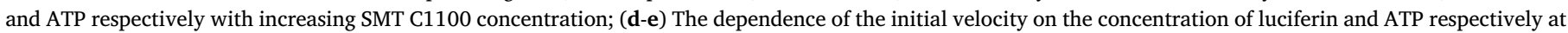

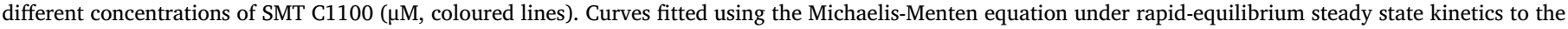
data. Decrease in $\mathrm{V}_{\max }$ at $500 \mu \mathrm{M}$ luciferin $\left(\mathrm{D}-\mathrm{LH}_{2}\right.$ ) is caused by substrate inhibition [63].

SMT C1100's mode of inhibition is similar to that of other reported noncompetitive inhibitors, resveratrol 11, NF-kBAI4 12 and compound 15 were included in further experiments (Supplementary Fig. S4). Resveratrol and structurally related $\mathbf{1 2}$ were found to have non-competitive and partial non-competitive inhibition respectively regarding $\mathrm{LH}_{2}$ AMP, whilst 15 had mixed partial inhibition similar to SMT C1100 (table, Fig. 4c). These findings indicate that these non-competitive inhibitors are binding via a previously undescribed mechanism. This indicates that FLuc has multiple inhibitor binding sites and conformations beyond that which is currently understood.

$\mathrm{X}$-ray crystallography is a powerful method for determining proteinligand binding modes. Co-crystallisation of FLuc with SMT C1100 alone or in the presence of ATP, luciferin or DLSA was attempted in this work to determine the exact location of the SMT C1100 binding site. However crystallisation attempts yielded only apo structures matching those previously reported in the literature $[30,31]$. This suggests that SMT C1100 may bind to a higher energy conformation of FLuc which we have been unable to crystallise.

\subsection{Design and synthesis of a photoaffinity probe}

In the absence of X-ray crystal data, a photoaffinity strategy was employed to elucidate the binding mode of SMT C1100. This approach involves incorporation of a photoaffinity group into a probe compound then exploiting its affinity for its protein target (Fig. 5b). Once bound,
UV irradiation to activate the photoaffinity group results in carbene generation, then covalent binding to nearby residues [48]. The site of incorporation can be established by mass spectrometry to reveal the molecule's binding site. A trifluoromethyl-phenyl diazirine (TPD) was selected for incorporation into SMT C1100's structure, due to its small size and expected small impact on FLuc binding affinity. Indeed, the sterics and electronics of the diazirine moiety appear a good match SMT C1100's sulfonyl group. Furthermore, TPDs are well-characterised and have been successfully used in the literature for binding site identification studies [49-51]. Photoaffinity probe 16 retained FLuc inhibition behaviour, although with a reduced affinity, $\mathrm{IC}_{50}=0.595 \pm 0.112 \mu \mathrm{M}$ (Supplementary Fig. S5).

\subsection{Oxidation of probe reveals an alternative mechanism}

However, incubation of the probe with FLuc, followed by UV irradiation $(365 \mathrm{~nm})$ and LC-MS of the reaction mixture resulted in no observation of probe incorporation into the enzyme (Fig. 5d). Instead, two species were generated from the probe, one whose mass corresponds to the addition of $\mathrm{H}_{2} \mathrm{O}_{2}$ assigned as hydrate 18 the other corresponding to the addition of water, assigned as 17 (Fig. 5a). This finding was also observed with the addition of substrates luciferin, ATP and DLSA. However, interestingly, when the probe was irradiated in the absence of FLuc (Fig. 5c), only the water adduct was observed, indicating the requirement of the enzyme for the oxidation of the probe. 
a

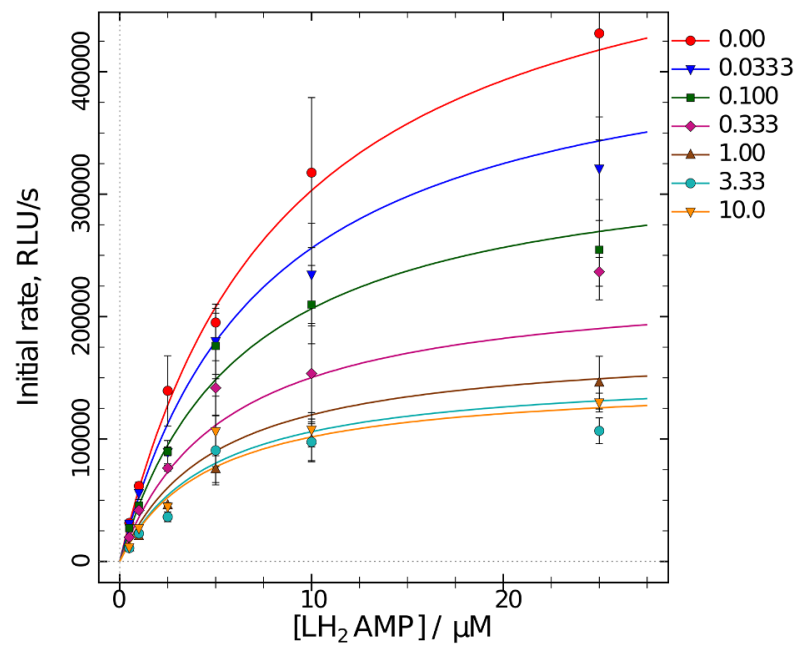

C b

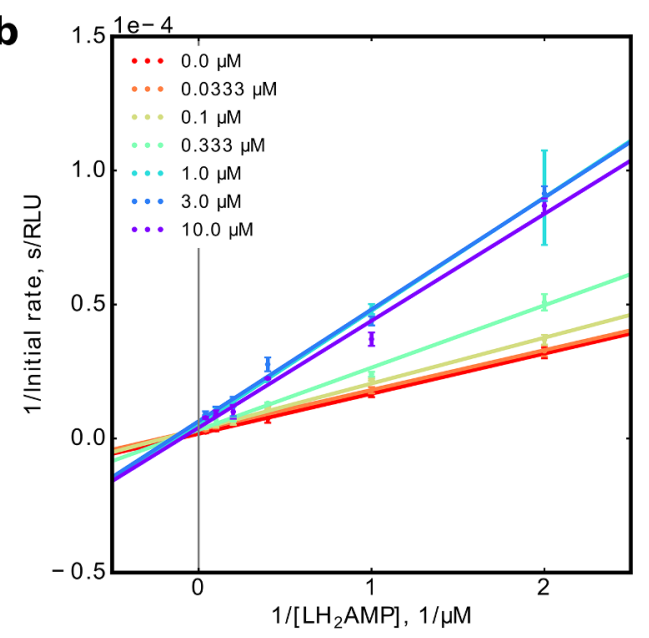
Ezutromid
DLSA
Resveratrol
NF-KBAI4
15

\begin{tabular}{rcccc|}
\hline K $\mathrm{K}_{\mathrm{M}}$ increased & $\mathrm{K}_{\mathrm{M}}$ increased & $\mathrm{K}_{\mathrm{M}}$ unchanged & $\mathrm{K}_{\mathrm{M}}$ unchanged & $\mathrm{K}_{\mathrm{M} \text { decreased }}$ \\
$\mathrm{V}_{\max }$ decreased & $\mathrm{V}_{\max }$ unchanged & $\mathrm{V}_{\max }$ decreased & $\mathrm{V}_{\max }$ decreased & $\mathrm{V}_{\max }$ decreased \\
E.S.I - E.I $+\mathrm{P}$ & E.S.I $\nrightarrow$ E.I $+\mathrm{P}$ & E.S.I $\nrightarrow$ E.I $+\mathrm{P}$ & E.S.I - E.I + P & E.S.I E.I + P \\
\hline Mixed partial & Competitive & Non-competitive & Partial non-competitive & Mixed partial \\
\hline
\end{tabular}

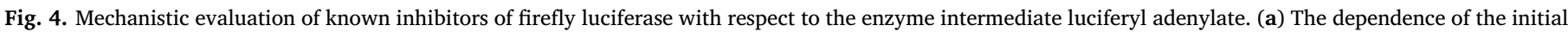

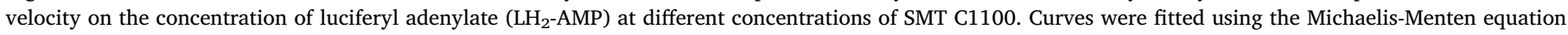

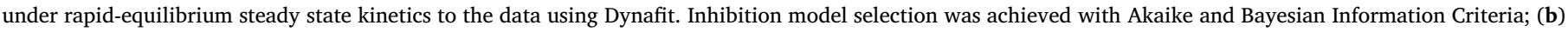

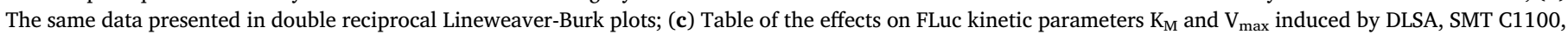

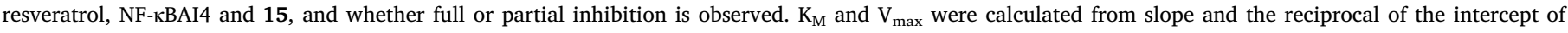

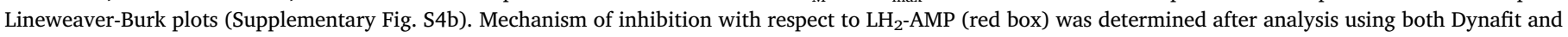
Lineweaver-Burk methods.

ESI MS/MS fingerprint analysis of the putative water 17 and $\mathrm{H}_{2} \mathrm{O}_{2} \mathbf{1 8}$ adducts generated in the irradiation experiments corresponded with the spectra generated from authentic samples of the compounds (Fig. 5e, synthesis described in the SI). Addition of molecular oxygen to TPDs has been reported in the literature [52] and FLuc has oxygenase activity (Fig. 1a), indeed an oxygen/pantetheine tunnel to the active site that has been postulated by Branchini et al. [53,54]. Furthermore, the approach of oxygen to the active site has been modelled by molecular dynamics and mechanics, finding that oxygen can move inside FLuc without any energetic barrier [55]. The results of this experiment suggest that probe $\mathbf{1 6}$ might bind to an oxygen-bound FLuc conformer.

\section{Conclusions}

SMT C1100, although shown to genuinely increase utrophin at the mRNA and protein levels in vitro and in vivo [42-44], has also been identified as an FLuc inhibitor, requiring cautious interpretation of results from its use in FLuc reporter gene assays. SMT C1100 is a noncompetitive inhibitor with respect to luciferin and uncompetitive with respect to ATP. SMT C1100, alongside three other literature non-competitive inhibitors, was found to have previously undescribed noncompetitive and mixed inhibition mechanisms with respect to the intermediate $\mathrm{LH}_{2}$-AMP. Photoirradiation studies to identify the binding site of a SMT C1100 probe revealed the formation of an FLuc-dependent oxidation product, consistent with binding of the probe in the proximity of molecular oxygen. However, the binding site of these, along with many other non-competitive inhibitors, is still uncharacterised. This along with a more detailed understanding of the FLuc catalytic mechanism and the dynamic conformational changes involved may form the basis of future studies.

\section{Experimental procedures}

\subsection{Materials}

The following materials were bought from commercial sources: resveratrol (Santa Cruz Biotechnology), NF-кB inhibitor NF-кBAI4 (Cambridge Bioscience Ltd), D-luciferin (Promega), ATP (Sigma), recombinant firefly luciferase (QuantiLum Promega, E1701). Ezutromid was provided by Summit Therapeutics plc.

Analytical procedures and chemical synthesis of $\mathrm{LH}_{2}$-AMP, DLSA, 15, 16, 17 and 18 are described in the supplementary information.

\subsection{Cell culture}

H2K-mdx utrnA-luc cells $[43,56]$ were maintained in DMEM (Life Technologies) supplemented with $20 \%$ Fetal Bovine Serum (Life Technologies), 2\% CEE (SLI), $2 \mathrm{mM}$ L-Glutamine (Life Technologies), $1 \%$ Penicillin Streptomycin (Life Technologies) and $2 \mu \mathrm{g} / 500 \mathrm{~mL}$ Mouse Interferon- $\gamma$ (Roche). Cells were maintained at $10 \% \mathrm{CO}_{2}$ at $33^{\circ} \mathrm{C}$. 


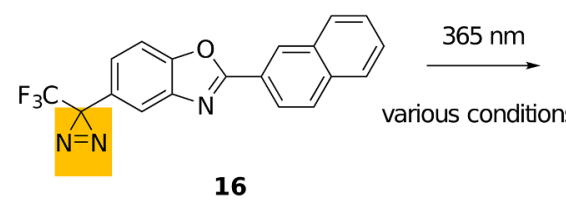

16

b

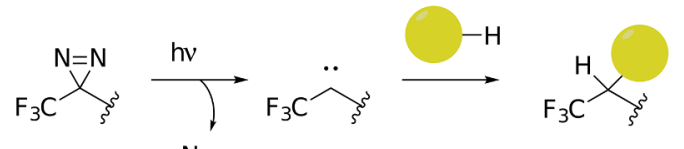

C

\begin{tabular}{|c|c|c|c|}
\hline FLuc & Substrate(s) & $\begin{array}{c}17 \\
\text { MW=344 }\end{array}$ & $\begin{array}{c}18 \\
\text { MW = 360 }\end{array}$ \\
\hline$\times$ & None & $\checkmark$ & $\times$ \\
\hline$\checkmark$ & None & $\checkmark$ & $\checkmark$ \\
\hline$\checkmark$ & D-Luciferin & $\checkmark$ & $\checkmark$ \\
\hline$\checkmark$ & ATP & $\checkmark$ & $\checkmark$ \\
\hline$\checkmark$ & DLSA & $\checkmark$ & $\checkmark$ \\
\hline$\checkmark$ & $\begin{array}{c}\text { D-Luciferin } \\
\text { and ATP }\end{array}$ & $\checkmark$ & $\checkmark$ \\
\hline
\end{tabular}
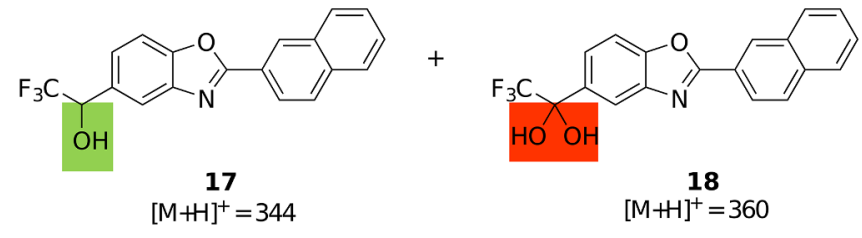

d

e

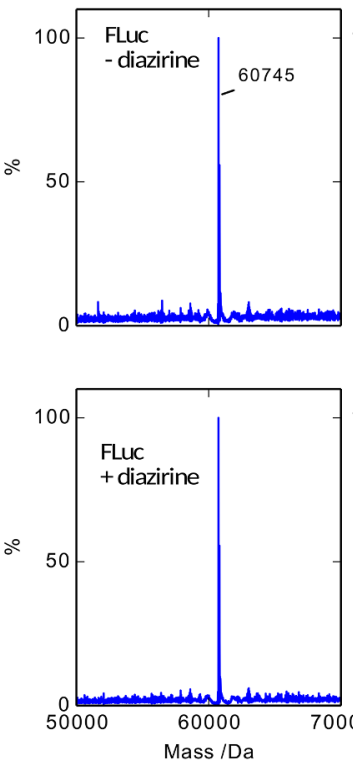

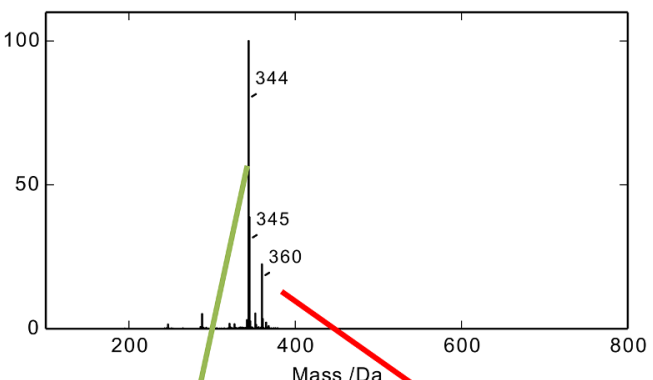

Fig. 5. Irradiation studies of SMT C1100-related photoaffinity probe with firefly luciferase. (a) Scheme of products generated from irradiation of diazirine probe $\mathbf{1 6}$ under conditions listed in table (c); (b) Schematic of photo-induced covalent crosslinking of trifluorophenyl diazirines into - $\mathrm{CH}$ or - $\mathrm{XH}$ where $\mathrm{X}$ is a heteroatom; (d) LC-MS of FLuc with (bottom) and without (top) diazirine probe 16; (e) LC-MS of products formed from diazirine irradiation, bottom left: MS/MS spectrum of peak 344, bottom right: MS/MS spectrum of peak 360.

\subsection{Utrophin-A FLuc reporter gene assay}

White flat bottomed 96 well plates (Corning) were seeded with 5000 $\mathrm{H} 2 \mathrm{~K}-m d x$ utrnA-luc cells. After $24 \mathrm{~h}$ at $10 \% \mathrm{CO}_{2}$ and $33^{\circ} \mathrm{C}$, the cells were dosed with compound in triplicate, in the following concentration series: $0.01,0.03,0.1,0.3,1.0,3.0,10.0 \mu \mathrm{M}$ from $10 \mathrm{mM}$ solution stocks in DMSO (final DMSO concentration was $0.3 \%$ ). The cells were incubated for a further $24 \mathrm{~h},\left(10 \% \mathrm{CO}_{2}, 33^{\circ} \mathrm{C}\right)$. Relative luminescence readout after using the Luciferase Assay System (Promega, E1500) reagents was measured using a FLUOstar Optima plate reader (BMG Labtech). The means from the biological triplicates were fitted with a four parameter logistic function with least squares regression (Levenberg-Marquardt algorithm) to calculate $\mathrm{EC}_{50}$ values.

\subsection{Biochemical FLuc inhibition assay}

Recombinant FLuc was assayed at a final concentration of $0.6 \mathrm{nM}$ in a buffer containing $25 \mathrm{mM}$ HEPES, $5 \mathrm{mM} \mathrm{MgCl}_{2}, 1 \mathrm{mM}$ EDTA, $5 \mathrm{mM}$ DTT and $1 \mathrm{mg} / \mathrm{ml} \mathrm{BSA}$. Compounds were diluted in the following concentration series: $0 \mathrm{nM}, 0.95 \mathrm{nM}, 3 \mathrm{nM}, 30 \mathrm{nM}, 95 \mathrm{nM}, 0.3 \mu \mathrm{M}$, $0.95 \mu \mathrm{M}, 3 \mu \mathrm{M}, 9.5 \mu \mathrm{M}, 30 \mu \mathrm{M}$, from $10 \mathrm{mM}$ stocks in DMSO (with a final assay concentration of DMSO at $0.3 \%$ ). PTC124 was used as a positive control [36]. Luciferase substrates ATP and D-luciferin were used in a final assay concentration of $10 \mu \mathrm{M}$. Luciferase was pre-incubated with ATP and the query compound at $0{ }^{\circ} \mathrm{C}$ for $15 \mathrm{~min}$. D-Luciferin was dispensed and endpoint luminescence output immediately read using a FLUOstar Optima plate reader (BMG Labtech). Luminescence output was fitted with a four parameter logistic function with least squares regression (Levenberg-Marquardt algorithm) to calculate $\mathrm{EC}_{50}$ values.

\subsection{FLuc fluorescence quenching assay}

Fluorescence quenching of SMT C1100 upon binding recombinant FLuc was monitored using a fluorescence spectrophotometer (Hitachi F4500 FL spectrophotometer, $317 \mathrm{~nm}$ excitation/390 nm emission). $0.3 \mu \mathrm{M}$ SMT C1100 was incubated with FLuc (various concentrations ranging $0-3 \mu \mathrm{M}$ ) for $15 \mathrm{~min}$ in a buffer containing $25 \mathrm{mM}$ HEPES, $5 \mathrm{mM} \mathrm{MgCl}_{2}, 1 \mathrm{mM}$ EDTA, $5 \mathrm{mM}$ DTT and 1\% DMSO. Fluorescence emission was measured in each sample and the $\mathrm{K}_{\mathrm{D}}$ calculated by fitting the curve with a four parameter logistic function.

\subsection{Docking of FLuc luciferin pocket}

Docking studies were carried out using AutoDock Vina using default settings [57]. The luciferin pocket of FLuc crystal structure PDB: 4e5d was prepared as follows: bound ligands were extracted using PyMOL, the protonation state relevant for $\mathrm{pH} 7.4$ was applied using a PDB2PQR $[58,59]$ server and the binding box was prepared using AutoDockTools4 [60]. Ligand files were prepared from sdf files with 3D coordinates generated using ChemAxon tools [61] then prepared for docking using AutoDockTools4.

\subsection{FLuc kinetics assay, varying ATP, luciferin or $\mathrm{LH}_{2}$-AMP as the sole substrate}

Recombinant FLuc was assayed at a final concentration of $0.6 \mathrm{nM}$ in a buffer containing $25 \mathrm{mM}$ HEPES, $5 \mathrm{mM} \mathrm{MgCl} 2,1 \mathrm{mM}$ EDTA, $5 \mathrm{mM}$ DTT and $1 \mathrm{mg} / \mathrm{ml}$ BSA. SMT C1100 was diluted in a concentration series from $10 \mathrm{mM}$ stocks in DMSO (with a final assay concentration of DMSO at $0.3 \%$ ). Either D-luciferin or ATP was varied in the following 
concentration series: $1 \mu \mathrm{M}, 5 \mu \mathrm{M}, 10 \mu \mathrm{M}, 50 \mu \mathrm{M}, 100 \mu \mathrm{M}, 500 \mu \mathrm{M}$ in triplicate, with the other substrate held at the saturating concentration of $250 \mu \mathrm{M}$ (added immediately prior to measuring luminescence output). For the $\mathrm{LH}_{2}$-AMP experiment, a concentration series of $0.5 \mu \mathrm{M}$, $1 \mu \mathrm{M}, 2.5 \mu \mathrm{M}, 5 \mu \mathrm{M}, 10 \mu \mathrm{M}, 25 \mu \mathrm{M} \mathrm{LH} \mathrm{LH}_{2}$-AMP was used and no other substrate was supplied. Luminescence output was measured every $0.04 \mathrm{~s}$ for the first $4 \mathrm{~s}$ of the reaction using a FLUOstar Optima plate reader (BMG Labtech) and the initial rate was calculated by linear slope fitting. The initial rates data were plotted and fitted using Dynafit4 for inhibition model discrimination by Akaike and Bayesian Information Criteria [62].

\subsection{FLuc diazirine irradiation study}

$20 \mu \mathrm{M}$ diazirine probe 16 was incubated with $10 \mu \mathrm{M}$ recombinant FLuc in a buffer containing $25 \mathrm{mM}$ HEPES, $5 \mathrm{mM} \mathrm{MgCl}_{2}, 1 \mathrm{mM}$ EDTA, $5 \mathrm{mM}$ DTT and 5\% DMSO. Where applicable, $100 \mu \mathrm{M}$ ATP, $50 \mu \mathrm{M}$ luciferin or $20 \mu \mathrm{M}$ DLSA were included. After 15 mins incubation at $0{ }^{\circ} \mathrm{C}$, the samples were irradiated ( $365 \mathrm{~nm}, 100 \mathrm{~W}$ UV lamp, $\left.\mathrm{UVP}^{\mathrm{TM}} \mathrm{B}-100 \mathrm{AP}\right)$ for 3 mins at $0{ }^{\circ} \mathrm{C}$ from a distance of $6 \mathrm{~cm}$. The samples were diluted 1:1 with milli-Q water and submitted for LC-MS analysis on a Waters Acquity $1525 \mu$ HPLC system coupled to a LCT Premier XE (Waters) mass spectrometer. A flow rate of $0.3-0.75 \mathrm{~mL} / \mathrm{min}$ was applied to a mobile phase of $\mathrm{A}=$ water $+0.1 \%$ formic acid, $\mathrm{B}=\mathrm{MeCN}$ with a gradient of \%A: $0.0 \mathrm{~min} 97 \%, 1.0 \mathrm{~min} 97 \%, 5.0 \mathrm{~min} 2 \%, 6.0 \mathrm{~min} 2 \%$, $7.0 \mathrm{~min} 97 \%, 10.0 \mathrm{~min} 97 \%$. The electrospray source had a capillary voltage of $3.00 \mathrm{kV}$ and cone voltage of $100 \mathrm{~V}$. Nitrogen was the nebuliser and desolvation gas, flow of $300 \mathrm{~L} / \mathrm{hr}$. Total mass spectra were deconvoluted using the MaxEnt algorithm in MassLynx 4.1 (Waters) according to the default settings.

ESI MS/MS analysis was carried out on an Acquity-UPLC system (Waters) connected to a Xevo G2-XS Q-TOF mass spectrometer (Waters) equipped with an electrospray ion source. The analyte separated on an ACE equivalent $3 \mathrm{C} 18$ analytical column $(2.1 \mathrm{~mm}$ i.d. $\times 50 \mathrm{~mm}, 3 \mu \mathrm{m}$, $100 \AA$ A) using a linear gradient (length: $10 \mathrm{~min}, 5 \%$ to $95 \%$ solvent $\mathrm{B}$ ( $0.1 \%$ formic acid in acetonitrile)) at a flow rate: $0.4 \mathrm{~mL} / \mathrm{min}$ (solvent A $0.1 \%$ formic acid in water). The separated peptides were electrosprayed directly into the mass spectrometer operating in a full scan method using a CID based method. A full scan MS spectrum was operated in electrospray positive mode with a scan range $100-900 \mathrm{~m} / \mathrm{z}$ and a scan time $1.5 \mathrm{~s}$. Lockspray was used during analysis to maintain mass accuracy. Data was obtained in continuum mode. The electrospray source of the MS was operated with a capillary voltage of $3.00 \mathrm{kV}$ and a cone voltage of $40 \mathrm{~V}$. CID fragmentation was performed at a ramp from 35 to $45 \mathrm{~V}$ of normalized collision energy. The MS and MS/MS analysis was processed using Masslynx. The mass window search of precursor ion was set as 5 ppm error. All spectra were manually checked and validated.

\section{Data availability statement}

All data generated or analysed during this study are included in this published article (and its Supplementary Information files).

\section{Acknowledgements}

This work was supported by funding from the Engineering and Physical Sciences Research Council (EPSRC) and the Medical Research Council (MRC) [grant number EP/L016044/1, I.V.L.W.], Summit Therapeutics [I.V.L.W., A.V. \& G.M.W.] and Muscular Dystrophy UK [grant number RA4/3013/4, A.V.].

\section{Author contributions statement}

I.V.L.W, S. R. G. and A. J. R. conceived and planned the experiments. J. K. R. synthesised the photoaffinity probe, A. J. S. and I. V. L.
W. synthesised DLSA. I.V.L.W. performed the experiments except the ESI MS/MS experiment which was run and analysed by E.P.. I.V.L.W. prepared the manuscript and figures. All authors reviewed the manuscript.

\section{Declaration of Competing Interest}

F.X.W. is employed by Summit Therapeutics plc. A.J.R. and G.M.W. are shareholders of Summit Therapeutics plc.

\section{Appendix A. Supplementary material}

Supplementary data to this article can be found online at https:// doi.org/10.1016/j.bioorg.2019.103395.

\section{References}

[1] N. Thorne, J. Inglese, D.S. Auld, Illuminating insights into firefly luciferase and other bioluminescent reporters used in chemical biology, Chem. Biol. 17 (2010) 646-657.

[2] F. Fan, K.V. Wood, Bioluminescent assays for high-throughput screening, Assay Drug Dev. Technol. 5 (2007) 127-136.

[3] J.F. Thompson, L.S. Hayes, D.B. Lloyd, Modulation of firefly luciferase stability and impact on studies of gene regulation, Gene 103 (1991) 171-177.

[4] N. Thorne, et al., Firefly luciferase in chemical biology: A compendium of inhibitors, mechanistic evaluation of chemotypes, and suggested use as a reporter, Chem. Biol. 19 (2012) 1060-1072.

[5] P. Dranchak, et al., Profile of the GSK published protein kinase inhibitor set across ATP-dependent and-independent luciferases: implications for reporter-gene assays, PLoS ONE 8 (2013) e57888.

[6] C.A. Lyssiotis, et al., Reprogramming of murine fibroblasts to induced pluripotent stem cells with chemical complementation of Klf4, Proc. Natl. Acad. Sci. 106 (2009) 8912-8917.

[7] I. Younis, et al., Rapid-response splicing reporter screens identify differential regulators of constitutive and alternative splicing, Mol. Cell. Biol. 30 (2010) 1718-1728.

[8] S. Rocha, K.J. Campbell, K.C. Roche, N.D. Perkins, The p53-inhibitor Pifithrin- $\alpha$ inhibits Firefly Luciferase activity in vivo and in vitro, BMC Mol. Biol. 4 (2003) 9.

[9] A. Bakhtiarova, et al., Resveratrol inhibits firefly luciferase, Biochem. Biophys. Res. Commun. 351 (2006) 481-484.

[10] T.T.Y. Wang, $\beta$-naphthoflavone, an inducer of xenobiotic metabolizing enzymes, inhibits firefly luciferase activity, Anal. Biochem. 304 (2002) 122-126.

[11] D.S. Auld, et al., Characterization of chemical libraries for luciferase inhibitory activity, J. Med. Chem. 51 (2008) 2372-2386.

[12] D.S. Auld, N. Thorne, D. Nguyen, J. Inglese, A specific mechanism for nonspecific activation in reporter-gene assays, ACS Chem. Biol. 3 (2008) 463-470.

[13] D.S. Auld, J. Inglese, Interferences with luciferase reporter enzymes assay guidance manual, Assay Guid. Man. [Internet] 1 (2016) 1-14.

[14] W.D. McElroy, A. Green, Function of adenosine triphosphate in the activation of luciferin, Arch. Biochem. Biophys. 64 (1956) 257-271.

[15] J.A. Sundlov, D.M. Fontaine, T.L. Southworth, B.R. Branchini, A.M. Gulick, Crystal structure of firefly luciferase in a second catalytic conformation supports a domain alternation mechanism, Biochemistry 51 (2012) 6493-6495.

[16] W.D. McElroy, H.H. Seliger, E.H. White, Mechanism of bioluminescence, chemiluminescence and enzyme function in the oxidation of firefly luciferin, Photochem. Photobiol. 10 (1969) 153-170.

[17] R. Fontes, A. Dukhovich, A. Sillero, M.A.G. Sillero, Synthesis of dehydroluciferin by firefly luciferase: Effect of dehydroluciferin, coenzyme a and nucleoside triphosphates on the luminescent reaction, Biochem. Biophys. Res. Commun. 237 (1997) $445-450$.

[18] P.K. Poutiainen, et al., Discovery of 5-benzyl-3-phenyl-4,5-dihydroisoxazoles and 5benzyl-3-phenyl-1,4,2-dioxazoles as Potent Firefly Luciferase Inhibitors, J. Med. Chem. 56 (2013) 1064-1073.

[19] J.M.M. Leitão, J.C.G. Esteves da Silva, Firefly luciferase inhibition, J. Photochem. Photobiol. B Biol. 101 (2010) 1-8.

[20] D.S. Auld, N. Thorne, W.F. Maguire, J. Inglese, Mechanism of PTC124 activity in cell-based luciferase assays of nonsense codon suppression, Proc. Natl. Acad. Sci. U. S. A. 106 (2009) 3585-3590.

[21] I. Ueda, H. Kamaya, Kinetic and thermodynamic aspects of the mechanism of general anesthesia in a model system of firefly luminescence in vitro, Anesthesiology 38 (1973) 425-436.

[22] J.M. Ignowski, D.V. Schaffer, Kinetic analysis and modeling of firefly luciferase as a quantitative reporter gene in live mammalian cells, Biotechnol. Bioeng. 86 (2004) $827-834$.

[23] K.A. Feeney, M. Putker, M. Brancaccio, J.S. O'Neill, In-depth characterization of firefly luciferase as a reporter of circadian gene expression in mammalian cells, $\mathrm{J}$. Biol. Rhythms 31 (2016) 540-550.

[24] M. Nakamura, et al., Firefly luciferase exhibits bimodal action depending on the luciferin chirality, Biochem. Biophys. Res. Commun. 331 (2005) 471-475.

[25] J.L. Denburg, R.T. Lee, W.D. McElroy, Substrate-binding properties of firefly 
luciferase: I. Luciferin-binding site, Arch. Biochem. Biophys. 134 (1969) 381-394.

[26] C. Ribeiro, J.C.G. Esteves da Silva, Kinetics of inhibition of firefly luciferase by oxyluciferin and dehydroluciferyl-adenylate, Photochem. Photobiol. Sci. 7 (2008) 1085.

[27] K. Takehara, H. Kamaya, I. Ueda, Inhibition of firefly luciferase by alkane analogues, Biochim. Biophys. Acta - Gen. Subj. 1721 (2005) 124-129.

[28] K. Niwa, Y. Ohmiya, Inhibitory effect of lipoic acid on firefly luciferase bioluminescence, Biochem. Biophys. Res. Commun. 323 (2004) 625-629.

[29] H. Bai, et al., Discovery of a series of 2-phenylnaphthalenes as firefly luciferase inhibitors, RSC Adv. 5 (2015) 63450-63457.

[30] N.P. Franks, A. Jenkins, E. Conti, W.R. Lieb, P. Brick, Structural basis for the inhibition of firefly luciferase by a general anesthetic, Biophys. J. 75 (1998) 2205-2211.

[31] H. Zhang, et al., Inhibiting firefly bioluminescence by chalcones, Anal. Chem. 89 (2017) 6099-6105.

[32] F. Chen, et al., Prediction of luciferase inhibitors by the high-performance MIECGBDT approach based on interaction energetic patterns, Phys. Chem. Chem. Phys. 19 (2017) 10163-10176.

[33] D. Ghosh, U. Koch, K. Hadian, M. Sattler, I.V. Tetko, Luciferase advisor: high-accuracy model to flag false positive hits in luciferase HTS assays, J. Chem. Inf. Model. 58 (2018) 933-942.

[34] A.D. Broom, Rational design of enzyme inhibitors: multisubstrate analog inhibitors, J. Med. Chem. 32 (1989) 2-7.

[35] P.B. Le Calvez, C.J. Scott, M.E. Migaud, Multisubstrate adduct inhibitors: Drug design and biological tools, J. Enzyme Inhib. Med. Chem. 24 (2009) 1291-1318.

[36] D.S. Auld, et al., Molecular basis for the high-affinity binding and stabilization of firefly luciferase by PTC124, Proc. Natl. Acad. Sci. 107 (2010) 4878-4883.

[37] Translarna | European Medicines Agency. Available at: https://www.ema.europa. eu/en/medicines/human/EPAR/translarna. (Accessed: 14th January 2019).

[38] E.M. Welch, et al., PTC124 targets genetic disorders caused by nonsense mutations, Nature 447 (2007) 87-91.

[39] A. Braeuning, S. Vetter, The nuclear factor $\kappa B$ inhibitor (E)-2-fluoro-4'-methoxystilbene inhibits firefly luciferase, Biosci. Rep. 32 (2012) 531-537.

[40] A. Braeuning, Firefly luciferase inhibition: a widely neglected problem, Arch. Toxicol. 89 (2015) 141-142.

[41] B.R. Branchini, M.H. Murtiashaw, J.N. Carmody, E.E. Mygatt, T.L. Southworth, Synthesis of an N-acyl sulfamate analog of luciferyl-AMP: A stable and potent inhibitor of firefly luciferase, Bioorganic Med. Chem. Lett. 15 (2005) 3860-3864.

[42] D.R. Chancellor, et al., Discovery of 2-arylbenzoxazoles as upregulators of utrophin production for the treatment of duchenne muscular dystrophy, J. Med. Chem. 54 (2011) 3241-3250.

[43] J.M. Tinsley, et al., Daily treatment with SMTC1100, a novel small molecule utrophin upregulator, dramatically reduces the dystrophic symptoms in the mdx mouse, PLoS ONE 6 (2011) e19189.

[44] S. Guiraud, D. Roblin, K.E. Davies, The potential of utrophin modulators for the treatment of Duchenne muscular dystrophy, Expert Opin. Orphan Drugs 6 (2018)
179-192.

[45] B.Y. Feng, B.K. Shoichet, A detergent-based assay for the detection of promiscuous inhibitors, Nat. Protoc. 1 (2006) 550-553.

[46] H. Bai, et al., Synthesis and biological evaluation of a series of aryl triazoles as firefly luciferase inhibitors, Medchemcomm 6 (2015) 418-424.

[47] Y. Liu, et al., Identification and synthesis of substituted pyrrolo[2,3-d]pyrimidines as novel firefly luciferase inhibitors, Bioorg. Med. Chem. 20 (2012) 5473-5482.

[48] A.E. Ruoho, H. Kiefer, P.E. Roeder, S.J. Singer, The mechanism of photoaffinity labeling, Proc. Natl. Acad. Sci. U. S. A. 70 (1973) 2567-2571.

[49] J. Brunner, H. Senn, F.M. Richards, 3-Trifluoromethyl-3-phenyldiazirine. A new carbene generating group for photolabeling reagents, J. Biol. Chem. 255 (1980) 3313-3318.

[50] T. Seifert, et al., Identification of the Binding Site of Chroman-4-one-Based Sirtuin 2-Selective Inhibitors using Photoaffinity Labeling in Combination with Tandem Mass Spectrometry, J. Med. Chem. 59 (2016) 10794-10799.

[51] H. Muranaka, T. Momose, C. Handa, T. Ozawa, Photoaffinity labeling of the human $\mathrm{A}_{2 \mathrm{~A}}$ adenosine receptor and cross-link position analysis by mass spectrometry, ACS Med. Chem. Lett. 8 (2017) 660-665.

[52] M. Nassal, 4'-(1-Azi-2,2,2-trifluoroethyl)phenylalanine, a photolabile carbene-generating analog of phenylalanine, J. Am. Chem. Soc. 106 (1984) 7540-7545.

[53] B.R. Branchini, et al., Mutagenesis evidence that the partial reactions of firefly bioluminescence are catalyzed by different conformations of the luciferase Cterminal domain, Biochemistry 44 (2005) 1385-1393.

[54] B.R. Branchini, et al., Experimental support for a single electron-transfer oxidation mechanism in firefly bioluminescence, J. Am. Chem. Soc. 137 (2015) 7592-7595.

[55] R. Berraud-Pache, R. Lindh, I. Navizet, QM/MM study of the formation of the dioxetanone ring in fireflies through a superoxide ion, J. Phys. Chem. B 122 (2018) 5173-5182.

[56] Graham M. Wynne, et al., Benzoxazole benzimidazole and benzothiazole derivatives and their use in the treatment of Duchenne, Muscular Dystrophy (2009).

[57] O. Trott, A.J. Olson, AutoDock Vina: improving the speed and accuracy of docking with a new scoring function, efficient optimization, and multithreading, J. Comput. Chem. 31 (2010) 455-461.

[58] T.J. Dolinsky, J.E. Nielsen, J.A. McCammon, N.A. Baker, PDB2PQR: An automated pipeline for the setup of Poisson-Boltzmann electrostatics calculations, Nucleic Acids Res. 32 (2004).

[59] T.J. Dolinsky, et al., PDB2PQR: Expanding and upgrading automated preparation of biomolecular structures for molecular simulations, Nucleic Acids Res. 35 (2007).

[60] G.M. Morris, et al., AutoDock4 and AutoDockTools4: Automated docking with selective receptor flexibility, J. Comput. Chem. 30 (2009) 2785-2791.

[61] Marvin 16.16.6.0. marvin. ChemAxon (http://www.chemaxon.com).

[62] P. Kuzmič, Program DYNAFIT for the analysis of enzyme kinetic data: application to HIV proteinase, Anal. Biochem. 237 (1996) 260-273.

[63] N. Lembert, L.A. Idahl, Regulatory effects of ATP and luciferin on firefly luciferase activity, Biochem. J. 305 (Pt 3) (1995) 929-933. 